\title{
MCP-1 contributes to macrophage infiltration into adipose tissue, insulin resistance, and hepatic steatosis in obesity
}

\author{
Hajime Kanda, ${ }^{1}$ Sanshiro Tateya, ${ }^{1}$ Yoshikazu Tamori, ${ }^{1}$ Ko Kotani,, ${ }^{1}$ Ken-ichi Hiasa, ${ }^{2}$ Riko Kitazawa, ${ }^{3}$ \\ Sohei Kitazawa, ${ }^{3}$ Hitoshi Miyachi, ${ }^{4}$ Sakan Maeda, ${ }^{3}$ Kensuke Egashira, $^{2}$ and Masato Kasuga ${ }^{1}$

\begin{abstract}
1Department of Clinical Molecular Medicine, Kobe University Graduate School of Medicine, Kobe, Japan.
2Department of Cardiovascular Medicine, Graduate School of Medical Sciences, Kyushu University, Fukuoka, Japan.

${ }^{3}$ Department of Biomedical Informatics, Kobe University Graduate School of Medicine, Kobe, Japan. ${ }^{4}$ Laboratory for Animal Resources and Genetic Engineering, Center for Developmental Biology (CDB), Institute of Physical and Chemical Research (RIKEN), Kobe, Japan.
\end{abstract}

\begin{abstract}
Adipocytes secrete a variety of bioactive molecules that affect the insulin sensitivity of other tissues. We now show that the abundance of monocyte chemoattractant protein-1 (MCP-1) mRNA in adipose tissue and the plasma concentration of MCP-1 were increased both in genetically obese diabetic $(\mathrm{db} / \mathrm{db})$ mice and in WT mice with obesity induced by a high-fat diet. Mice engineered to express an MCP-1 transgene in adipose tissue under the control of the aP2 gene promoter exhibited insulin resistance, macrophage infiltration into adipose tissue, and increased hepatic triglyceride content. Furthermore, insulin resistance, hepatic steatosis, and macrophage accumulation in adipose tissue induced by a high-fat diet were reduced extensively in MCP-1 homozygous KO mice compared with WT animals. Finally, acute expression of a dominant-negative mutant of MCP-1 ameliorated insulin resistance in $\mathrm{db} / \mathrm{db}$ mice and in WT mice fed a high-fat diet. These findings suggest that an increase in $M C P-1$ expression in adipose tissue contributes to the macrophage infiltration into this tissue, insulin resistance, and hepatic steatosis associated with obesity in mice.
\end{abstract}

\section{Introduction}

Recent changes in human lifestyle have resulted in a marked increase in the incidence of obesity. Increased adiposity contributes to insulin resistance, dyslipidemia, hyperglycemia, and hypertension $(1,2)$. These pathological features are collectively referred to as metabolic syndrome, with insulin resistance being especially important in the pathogenesis of this state. The molecular mechanisms that link obesity and insulin resistance have thus been the subject of intensive investigation, although they remain incompletely understood.

Adipocytes are insulin-sensitive cells that take up glucose and store energy in the form of triglycerides, which are subsequently broken down to FFAs and glycerol in times of energy need. In addition to their storage function, adipocytes have recently been shown to be dynamic endocrine cells that produce and secrete various bioactive molecules (known as adipokines or adipocytokines), some of which affect the insulin sensitivity of other tissues, including the liver, skeletal muscle, pancreatic islets ( $\beta$ cells), and central nervous system. TNF- $\alpha$, leptin, plasminogen activator inhibitor-1, IL-6, resistin, and adiponectin have all been identified as adipokines (3). The insulin resistance that accompanies obesity is attributable, at least in part, to changes in the secretion of adipokines.

Monocyte chemoattractant protein-1 (MCP-1) is produced predominantly by macrophages and endothelial cells and is a potent chemotactic factor for monocytes (4-6). Expression of this proinflammatory chemokine is increased in atherosclerotic lesions

Nonstandard abbreviations used: CCR, CC chemokine receptor; G6Pase, glucose-6phosphatase; MCP, monocyte chemoattractant protein; 7ND, MCP-1 mutant lacking the aminoterminal 7 amino acids; PEPCK, phosphoenolpyruvate carboxykinase; SVF, stromal-vascular fraction.

Conflict of interest: The authors have declared that no conflict of interest exists. Citation for this article: J. Clin. Invest. 116:1494-1505 (2006). doi:10.1172/JCI26498.
$(7,8)$, and inhibition of its expression or that of its receptor, CC chemokine receptor 2 (CCR2), reduces the extent of atheroma formation in hypercholesterolemic mice $(9,10)$. These observations indicate that MCP-1 plays an important role in atherogenesis. In addition, the abundance of $M C P-1$ in both white adipose tissue and plasma is increased in obese mice (11), suggesting that MCP-1 might also be an adipokine whose expression is increased in obesity. However, to our knowledge a role for MCP-1 in obesity-induced insulin resistance has not been previously demonstrated.

In addition to that of MCP-1, the expression of several inflammation-related proteins, including TNF- $\alpha$, IL- 6 , and plasminogen activator inhibitor-1, in adipose tissue is increased in association with obesity (11-14). Furthermore, recent studies have demonstrated macrophage infiltration into adipose tissue of obese mice $(15,16)$. These observations suggest that adipose tissue in obesity is characterized by chronic low-grade inflammation that might contribute to the insulin resistance that accompanies this condition. We have now investigated the possible role of MCP- 1 in obesity-induced macrophage infiltration into adipose tissue and insulin resistance by generating mice with a gain or loss of function of MCP-1. The data we present here demonstrate that an increase in MCP-1 expression in adipose tissue contributes to the macrophage infiltration into this tissue, insulin resistance, and hepatic steatosis associated with obesity in mice.

\section{Results}

Identification of MCP-1 as a secretory factor of adipocytes. Mice that lack the glucose transporter GLUT4 specifically in adipocytes manifest insulin resistance in remote organs such as the liver and skeletal muscle (17), suggesting that adipocytes deficient in GLUT4-mediated glucose uptake might secrete a factor (or factors) that induces insulin resistance in other organs. To identify such a factor, we performed 
A

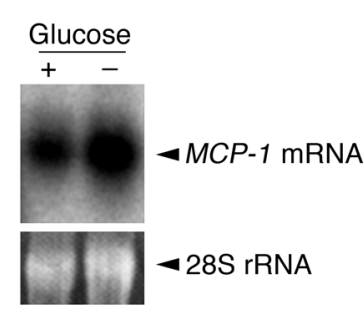

B

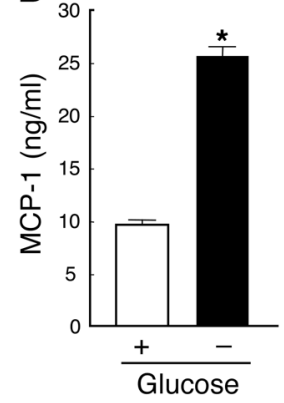

\section{Figure 1}

Upregulation of MCP-1 expression in 3T3-L1 adipocytes by glucose deprivation. (A) Total RNA $(15 \mu \mathrm{g})$ extracted from 3T3-L1 adipocytes cultured with or without glucose for 36 hours was subjected to Northern blot analysis with a probe specific for mouse MCP-1 mRNA. The region of the ethidium bromide-stained gel containing $28 S$ rRNA is also shown. (B) The culture supernatants of 3T3-L1 adipocytes cultured with or without glucose for 36 hours were assayed for MCP-1. Data are mean $\pm \operatorname{SEM}(n=18) .{ }^{\star} P<0.01$ versus culture with glucose.

microarray analysis with total RNA isolated from 3T3-L1 adipocytes after culture with or without glucose for 36 hours, with refreshment of the medium every 6 hours to prevent glucose depletion in the glucose-containing medium. Of the approximately 12,000 genes represented on the array, 43 showed a marked increase in expression in the cells deprived of glucose (data not shown). Eight of these 43 genes were predicted to encode secreted proteins on the basis of the presence of a signal peptide sequence. These 8 genes included those for $M C P-1$ and $M C P-3$, but the products of the remaining 6 genes were not known to play any role in glucose or lipid metabolism. Given that expression of the gene for MCP-1, but not for MCP-3, was substantially reduced after treatment of 3T3-L1 adipocytes for 36 hours with the thiazolidinedione rosiglitazone $(5 \mu \mathrm{M})$, we selected MCP-1 as the most promising candidate for a new adipokine that might induce insulin resistance. Retinol binding protein-4 (RBP4) was recently identified as an insulin resistance-inducing adipokine released from adipose tissue of adipocyte-specific GLUT4 KO mice (18). However, the RBP4 gene was not among the 43 genes whose expression level was shown by our microarray analysis to be significantly increased in 3T3-L1 adipocytes in response to glucose deprivation, since RBP4 was not expressed in 3T3-L1 adipocytes.

Our microarray results were confirmed by Northern blot analysis showing that the abundance of MCP-1 mRNA in 3T3-L1 adipocytes was greatly increased by glucose deprivation (Figure 1A). The concentration of MCP-1 in the culture supernatants of 3T3-L1 adipocytes was also increased significantly by glucose deprivation (Figure 1B). The presence of $M C P-1$ mRNA in 3T3-L1 adipocytes and the secretion of MCP-1 by these cells into the culture medium supported the notion that MCP- 1 is an adipokine. To examine the effect of hypoglycemia on MCP-1 secretion in vivo, we measured the plasma concentration of MCP-1 in mice deprived of food for 24 hours. However, we did not detect a significant difference in this parameter between fasted mice and control mice. The plasma glucose level of the fasted mice was approximately $60 \mathrm{mg} / \mathrm{dl}$, compared with a value of approximately $140 \mathrm{mg} / \mathrm{dl}$ for the control mice, suggesting that this level of hypoglycemia might not have been sufficient to increase MCP-1 secretion from adipocytes in vivo.

Upregulation of MCP-1 expression in obese mice. We next examined the tissue distribution of MCP-1 mRNA in obese mice. Northern blot analysis of total RNA extracted from various tissues of genetically obese diabetic $(\mathrm{db} / \mathrm{db})$ or lean control $(\mathrm{db} /+\mathrm{m})$ mice revealed the presence of a substantial amount of $M C P-1$ mRNA in white and brown adipose tissue of the former animals but not in that of the latter (Figure 2A). The plasma concentration of MCP-1 in $\mathrm{db} / \mathrm{db}$ mice was also about twice than of $\mathrm{db} /+\mathrm{m}$ mice (Figure $2 \mathrm{~B}$ ). Similarly, $M C P-1$ gene expression was detected in white and brown adipose tissue of mice with obesity induced by a high-fat diet but not in that of control mice fed normal chow (Figure 2C). Again, the plasma concentration of MCP-1 in mice fed the high-fat diet was about twice that of the control animals (Figure 2D). Furthermore, we directly compared the mRNA expression of $M C P-1$ in white adipose tissue on the same sheet in Northern blot analysis to avoid the difference of conditions in which Northern blot analysis was performed and confirmed that MCP-1 mRNA was actually increased in white adipose tissue of obese mice (Supplemental Figure 1; supplemental material available online with this article; doi:10.1172/ JCI26498DS1). These results indicate that obesity not only induces $M C P-1$ gene expression in adipose tissue but also increases the plasma concentration of this chemokine in mice. Our results are consistent with previous observations showing that the abundance of MCP- 1 mRNA in adipose tissue and the plasma concentration of MCP-1 are increased in obese mice $(11,15,16,19,20)$.

Histological analysis of white adipose tissue in obese mice. Obesity has been shown to be associated with a chronic low-grade inflammatory response in adipose tissue that is characterized by macrophage infiltration $(15,16)$. We therefore next examined white adipose tissue from $\mathrm{db} / \mathrm{db}$ mice and from mice fed a high-fat diet by immunohistochemical analysis with a $\mathrm{mAb}$ to $\mathrm{Mac3}$, a marker specific for mature macrophages. The antibody detected large multinucleate cells (accumulated macrophages) in epididymal adipose tissue from $\mathrm{db} / \mathrm{db}$ mice but not $\mathrm{db} /+\mathrm{m}$ mice (Supplemental Figure $2 \mathrm{~A}$ ). Macrophages were also detected in the adipose tissue of mice with obesity induced by a high-fat diet but were only rarely present in that of mice fed normal chow (Supplemental Figure 2B). The macrophages detected in mice on the high-fat diet were mononucleate or oligonucleate; multinucleate giant cells similar to those apparent in $\mathrm{db} / \mathrm{db}$ mice were rarely detected. We counted the number of infiltrated macrophages in adipose tissue by immunohistochemical staining and quantitated the degree of macrophage infiltration by calculating the ratio of infiltrated macrophages to total cells in adipose tissue (15). This analysis revealed that macrophage infiltration was actually increased significantly in both $\mathrm{db} / \mathrm{db}$ mice (Supplemental Figure 2C) and mice fed the high-fat diet (Supplemental Figure 2D) compared with their respective controls. Furthermore, the degree of macrophage infiltration in adipose tissue was significantly greater in $\mathrm{db} / \mathrm{db}$ mice than in mice fed the high-fat diet (17.7\% versus $13.1 \%$; Supplemental Figure 2, C and D). We also quantified the number of macrophages in the stromal-vascular fraction (SVF) of epididymal tissue of obese mice by flow cytometry after labeling of the cells with antibodies to both CD11b (macrophage marker) and CD45 (pan leukocyte marker). The percentage of double-positive cells (i.e., macrophages) in the SVF was significantly greater for $\mathrm{db} / \mathrm{db}$ mice than for $\mathrm{db} /+\mathrm{m}$ mice (17.1\% versus 3.6\%; Supplemental Figure $2 \mathrm{E}$ ) as well as for mice fed a high-fat diet compared with those fed normal chow (12.9\% versus 4.8\%; Supplemental Figure 2F).

Northern blot analysis revealed that the upregulation of $M C P-1$ mRNA in epididymal adipose tissue of $\mathrm{db} / \mathrm{db}$ mice and mice fed 

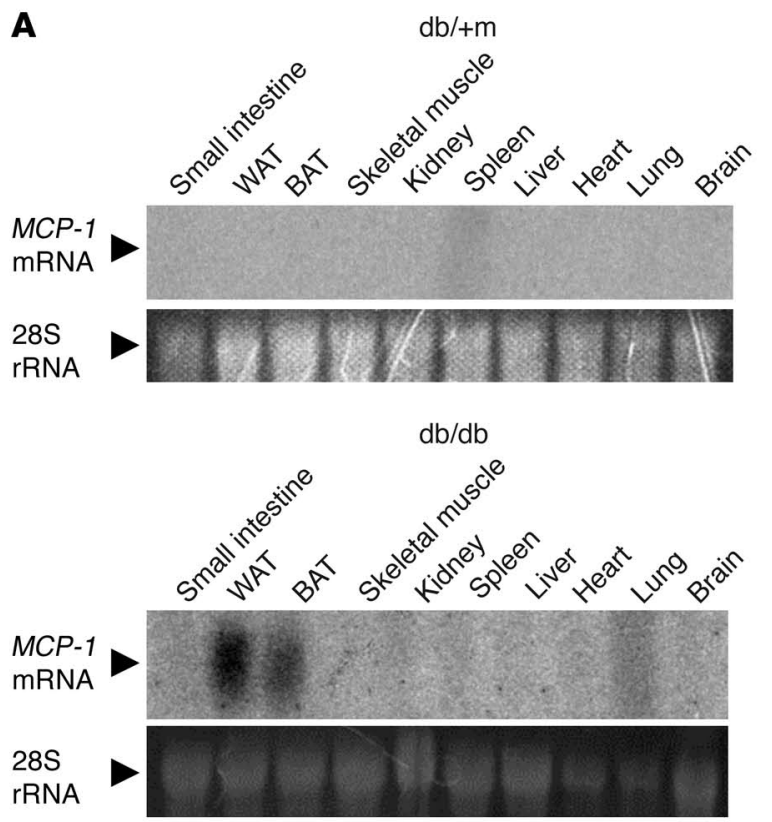

B

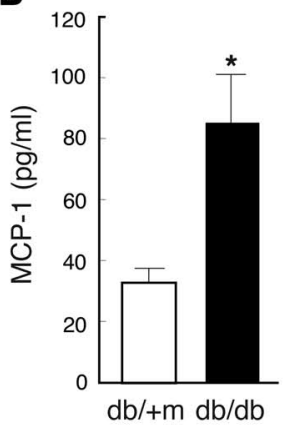

D

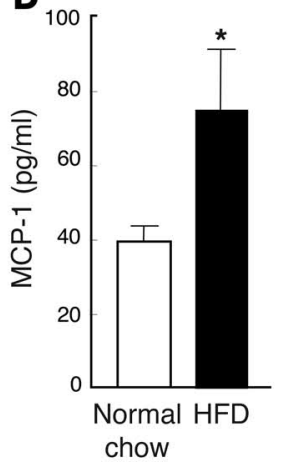

C

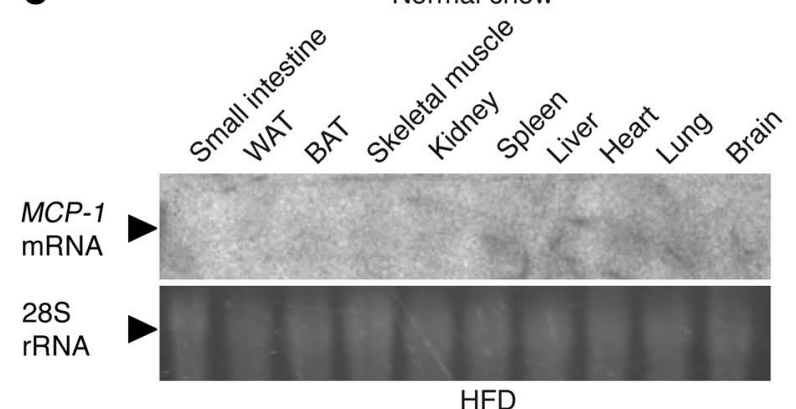

HFD

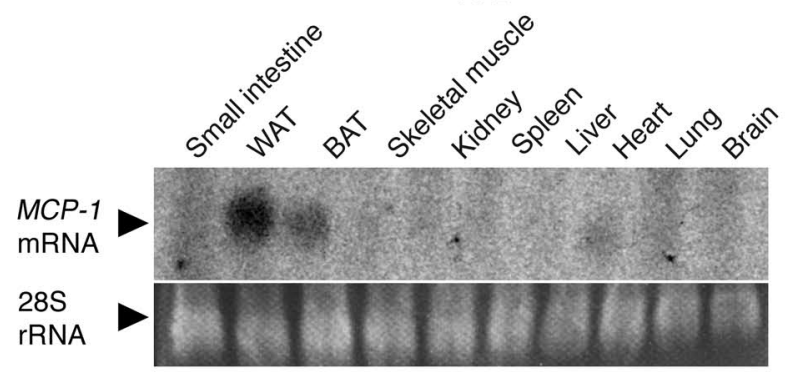

E
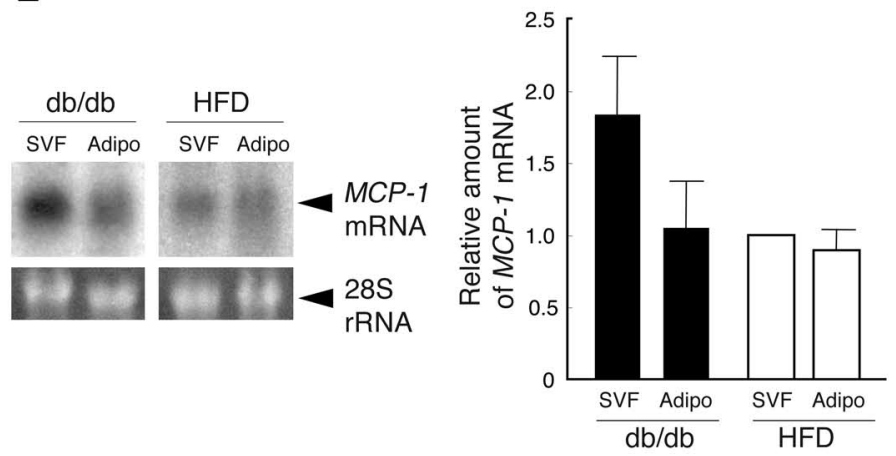

Figure 2

Tissue distribution of MCP-1 mRNA and plasma concentration of MCP-1 in obese mice. (A) Total RNA was extracted from the indicated tissues of 8 -week-old db/db or $\mathrm{db} /+\mathrm{m}$ mice and subjected to Northern blot analysis with a probe specific for mouse MCP-1 mRNA. WAT, white adipose tissue; BAT, brown adipose tissue. (B) Plasma concentration of MCP-1 in 11-week-old db/+m and db/db mice. Data are mean \pm SEM (db/+m, $n=8$; $\mathrm{db} / \mathrm{db}, n=11) .{ }^{*} P<0.05$ versus $\mathrm{db} /+\mathrm{m}$. (C) Total RNA, extracted from the indicated tissues of 18 -week-old C57BL/6J mice fed either a high-fat diet (HFD) or normal chow for 12 weeks, was subjected to Northern blot analysis with a probe specific for MCP-1 mRNA. (D) Plasma concentration of MCP-1 in 18-week-old C57BL/6J mice fed normal chow or the high-fat diet for 12 weeks. Data are mean \pm SEM (normal chow, $n=11$; high-fat diet, $n=9)$. ${ }^{*} P<0.05$ versus normal chow. (E) Total RNA $(15 \mu \mathrm{g})$, extracted from the SVF and adipocyte fraction (Adipo) of epididymal fat tissue from 11-week-old db/db mice or 18-week-old C57BL/6J mice fed a high-fat diet for 12 weeks, was subjected to Northern blot analysis with a probe specific for MCP-1 mRNA. The intensity of the band corresponding to MCP-1 mRNA in each fraction was quantitated and expressed relative to the value for the SVF of mice fed a high-fat diet. Data are mean \pm SEM of values from 3 independent experiments $(n=3)$.

a high-fat diet was apparent in both the adipocyte fraction and the SVF; it was more pronounced in the latter fraction in $\mathrm{db} / \mathrm{db}$ mice (Figure 2E). Together these results thus suggested that the increased abundance of MCP-1 mRNA in the SVF of adipose tissue in obese mice is attributable to infiltrated macrophages.

Insulin resistance, macrophage infiltration into adipose tissue, and increased hepatic triglyceride content in transgenic mice that overexpress $\mathrm{MCP}-1$ in adipocytes. We generated transgenic mice in which MCP-1 is overexpressed in adipocytes in order to investigate the primary effects of increased MCP-1 expression in adipose tissue. The transgene is composed of the mouse MCP-1 coding sequence under the control of the enhancer-promoter of the aP2 gene (21), which is active in brown and white adipocyte in vivo (22). We obtained 9 lines of transgenic mice with this construct, 2 of which (MCP-1 Tg-A and MCP-1 Tg-B) were characterized further.

Northern blot analysis confirmed that both the Tg-A (data not shown) and Tg-B (Figure 3A) lines expressed the transgene almost exclusively in adipose tissue. Furthermore, the plasma concentration of MCP-1 in MCP-1 Tg-B mice was increased approximately 3.9-fold compared with that in WT control animals (Figure 3B) and was approximately 1.6 times that of $\mathrm{db} / \mathrm{db}$ mice or mice fed a high-fat diet (compare Figure 2, B and D, with Figure $3 \mathrm{~B}$ ). Given that the plasma concentration of MCP-1 in MCP-1 Tg-A mice was increased approximately 100-fold compared with that in WT controls, we considered the MCP-1 Tg-B line to be a more acceptable model with which to examine the 
A
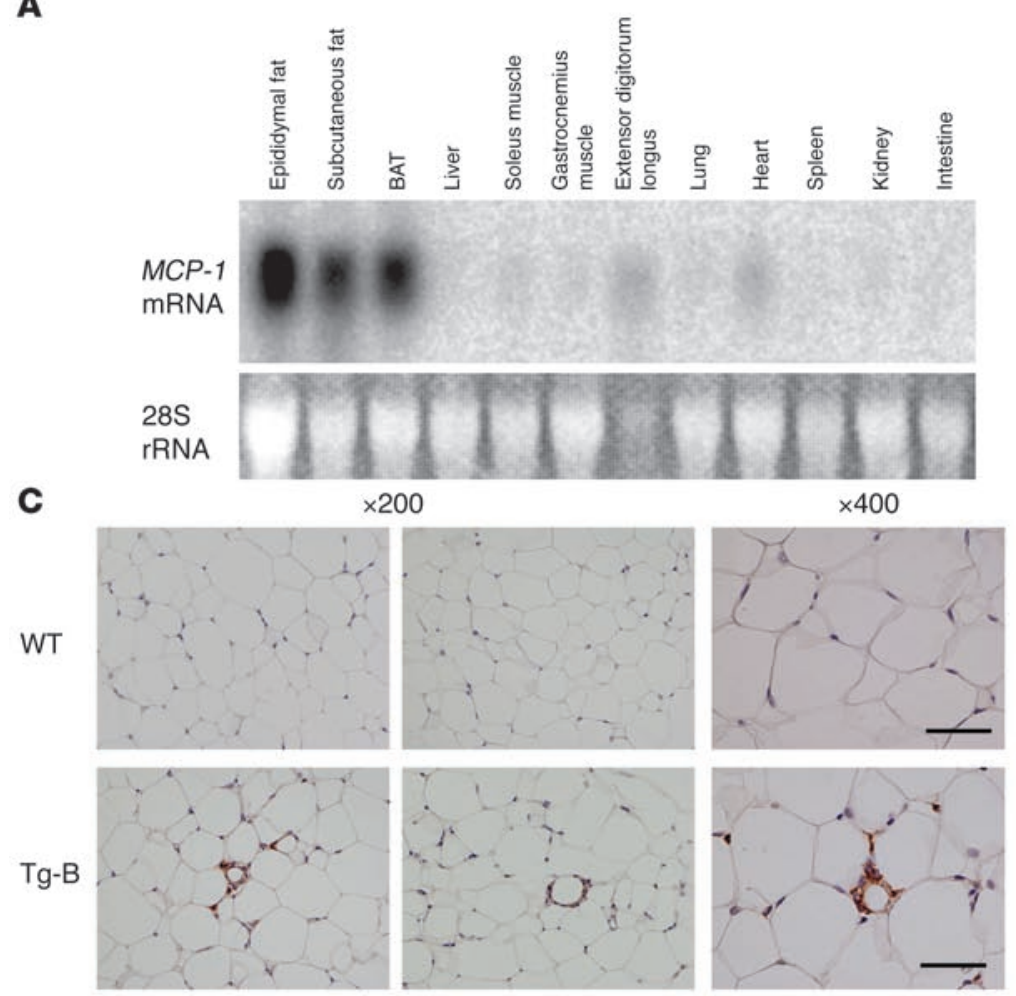

$\mathbf{F}$
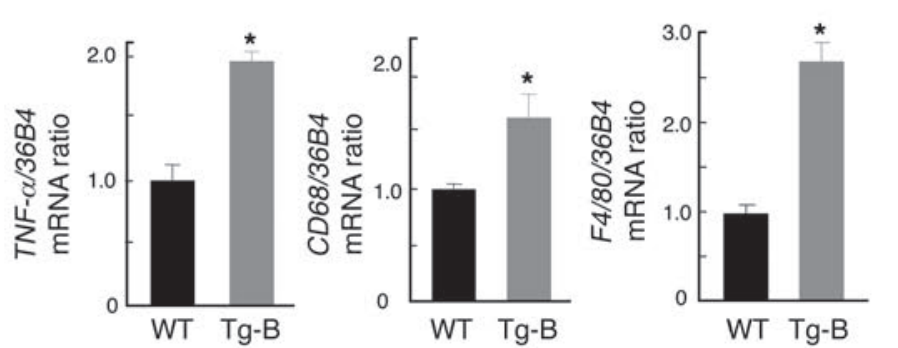

B

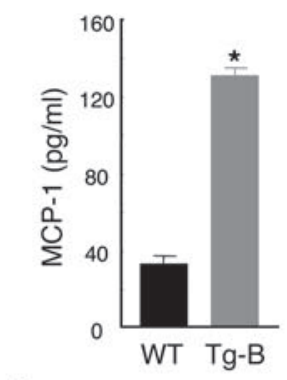

D
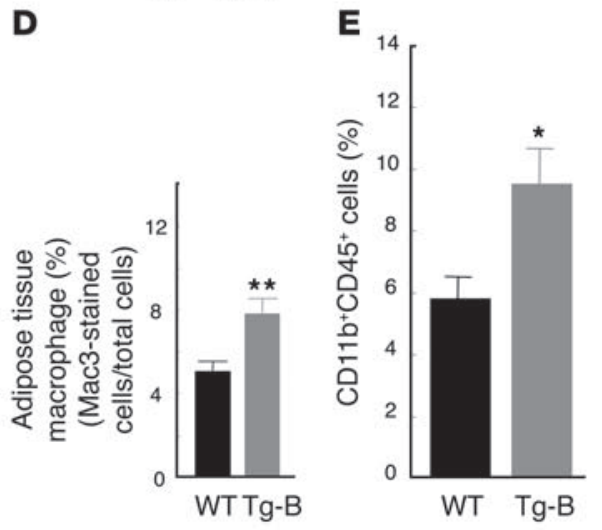

Figure 3

Generation and characterization of transgenic mice that overexpress MCP-1 in adipose tissue. (A) Northern blot analysis with an MCP-1 probe of total RNA isolated from various tissues of 11-week-old MCP-1 Tg-B mice. (B) Plasma concentration of MCP-1 in 13-week-old MCP-1 Tg-B and WT mice. Data are mean \pm SEM $(n=7)$. (C) Immunohistochemical detection of Mac3 in epididymal adipose tissue of 11-week-old MCP-1 Tg-B and WT mice. Macrophages are stained brown. Magnification, $\times 200$ and $\times 400$, as indicated. Scale bars: $50 \mu \mathrm{m}$. (D) Macrophage infiltration into epididymal fat tissue was quantitated in MCP-1 Tg-B $(n=7)$ and WT mice $(n=9)$ as the ratio of Mac3-positive cells to total cells. Data are mean \pm SEM. (E) Quantitation by flow cytometry of the proportion of CD11b+CD45+ cells (macrophages) in the SVF of epididymal fat tissue from 14-week-old MCP-1 Tg-B $(n=7)$ and WT mice $(n=6)$. Data are mean \pm SEM. (F) Quantitative RT-PCR analysis of total RNA isolated from epididymal fat tissue of 11 -week-old MCP-1 Tg-B and WT mice for TNF- $\alpha, C D 68$, and F4/80 mRNAs. Data (mean \pm SEM; $n=4$ ) were normalized by the amount of 36B4 mRNA and expressed relative to the corresponding WT value. ${ }^{*} P<0.05,{ }^{* *} P<0.01$ versus WT.

pathological role of increased MCP-1 expression in obese mice. We performed subsequent analyses with MCP-1 Tg-B mice and WT littermates as controls. MCP-1 Tg-B mice were viable and appeared normal overall.

Adipose tissue weight and adipocyte size did not differ significantly between MCP-1 Tg-B mice and WT controls (data not shown). Immunohistochemical analysis with the mAb to Mac3 revealed macrophage infiltration in white adipose tissue of MCP-1 Tg-B mice, whereas such infiltration was rarely detected in control animals (Figure 3, $\mathrm{C}$ and $\mathrm{D}$ ). The macrophages detected in adipose tissue of MCP-1 Tg-B mice were mononucleate or oligonucleate, similar to those detected in mice fed a high-fat diet. Flow cyto- metric analysis of macrophage infiltration into white adipose tissue with anti-CD11b and anti-CD45 revealed that the percentage of double-positive cells was increased significantly in Tg-B mice (Figure 3E). Macrophage infiltration in other insulin-responsive tissues, such as liver or skeletal muscle, of Tg-B mice was not evident by immunohistochemical analysis with anti-Mac3 (data not shown). In addition, quantitative RT-PCR analysis showed that the amounts of the mRNAs for TNF- $\alpha, C D 68$ (macrosialin), and F4/80 (a specific marker of mature macrophages) were significantly greater in the white adipose tissue of MCP-1 Tg-B mice than in that of control animals (Figure 3F), confirming the infiltration of macrophages into adipose tissue of the transgenic mice. 
A
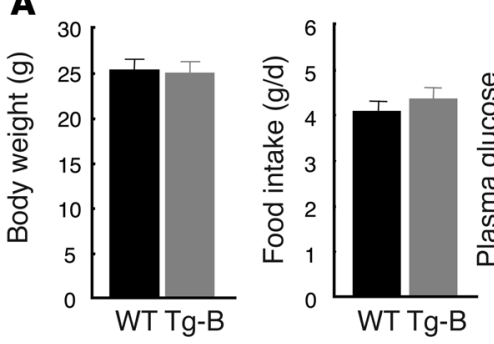

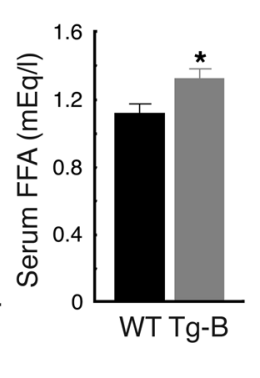

B

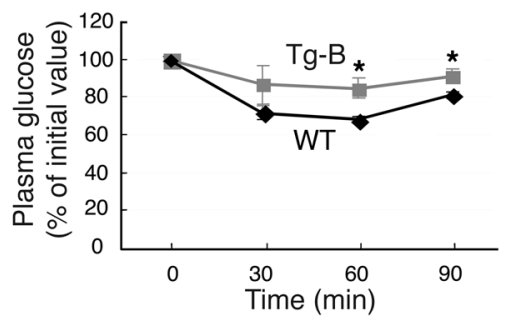

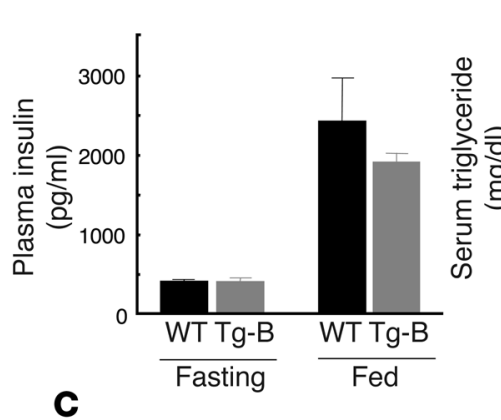

C

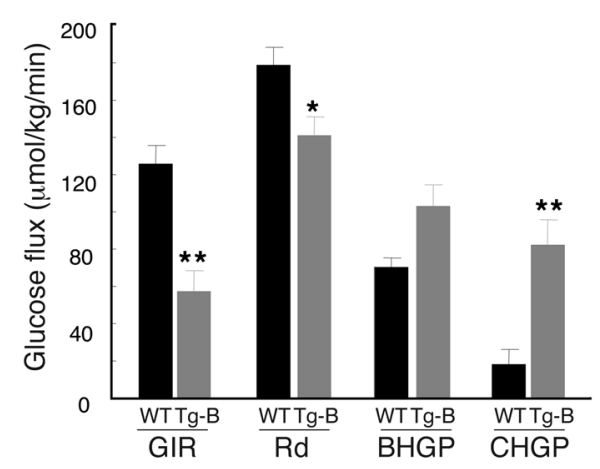

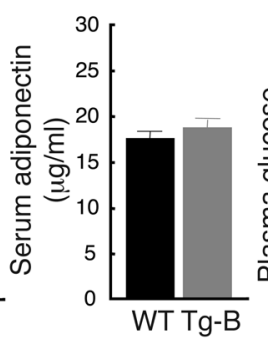
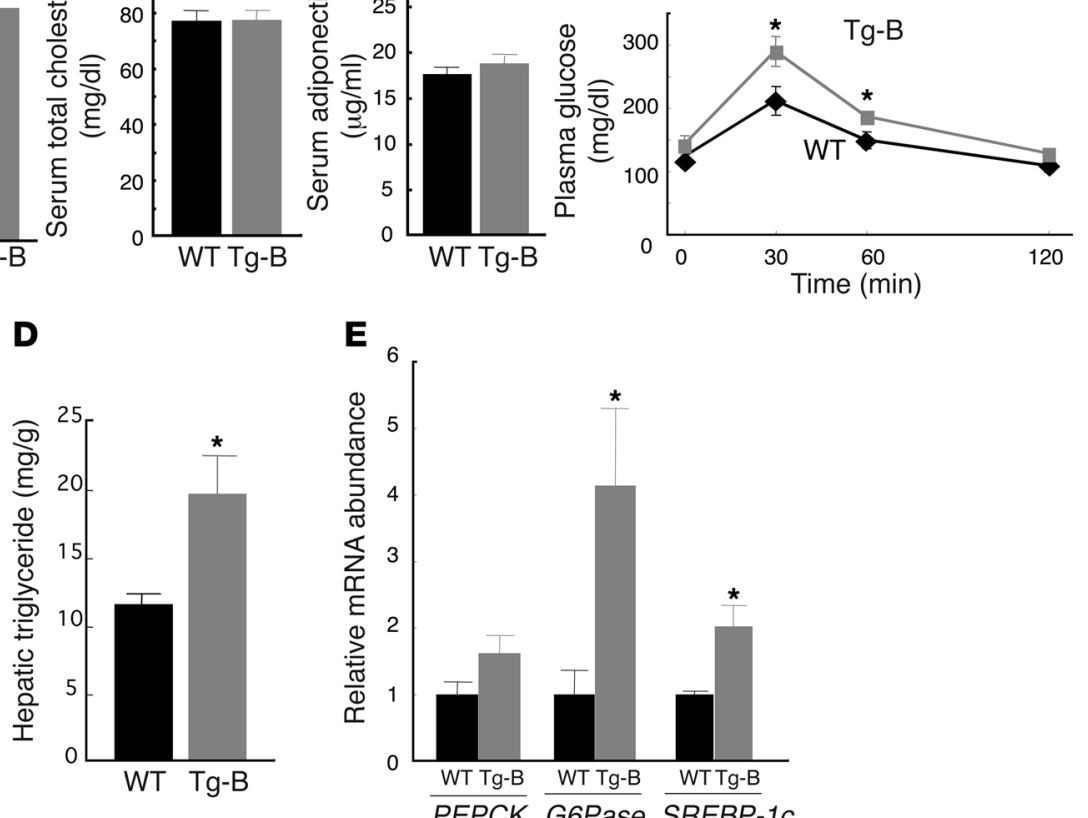

$\mathbf{E}$

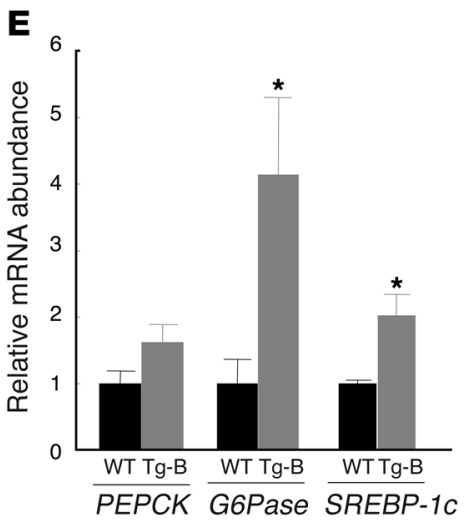

Figure 4

Metabolic characteristics of MCP-1 transgenic mice. (A) Metabolic parameters of 11-week-old MCP-1 Tg-B and WT mice. Data are means \pm SEM (body weight, $n=5$; food intake, $n=3$ [WT], 4 [Tg-B]; plasma glucose, $n=9$ [WT], 11 [Tg-B]; serum FFA, $n=9$ [WT], 11 [Tg-B]; plasma insulin, $n=5$; serum triglyceride, $n=4$ [WT], 6 [Tg-B]; serum total cholesterol, $n=9$ [WT], 11 [Tg-B]; serum adiponectin, $n=5$ ). (B) Plasma glucose level during insulin (upper panel) and glucose (lower panel) tolerance tests in WT and MCP-1 Tg-B mice at 12 to 13 weeks of age. Data are mean \pm SEM $(n=5)$. (C) Hyperinsulinemic-euglycemic clamp analysis in MCP-1 Tg-B $(n=5)$ and WT $(n=7)$ mice. Data are mean \pm SEM. GIR, glucose infusion rate; $\mathrm{Rd}$, rate of glucose disappearance; $\mathrm{BHGP}$, basal hepatic glucose production; CHGP, hepatic glucose production during clamp. (D) Hepatic triglyceride content of 11-week-old MCP-1 Tg-B and WT mice. Data are mean \pm SEM $(n=5)$. (E) Quantitation by Northern blot of the abundance of PEPCK, G6Pase, and SREBP-1c mRNAs in the liver of MCP-1 Tg-B and WT mice after hyperinsulinemic-euglycemic clamp analysis. Data (mean \pm SEM; PEPCK and SREBP-1C, $n=5[\mathrm{WT}], 4[\mathrm{Tg}-\mathrm{B}]$; G6Pase, $n=5$.) are expressed relative to the corresponding value for WT mice. ${ }^{*} P<0.05,{ }^{* *} P<0.01$ versus WT.

Body weight and food intake did not differ significantly between MCP-1 Tg-B mice and WT controls (Figure 4A). Although the plasma glucose levels of MCP-1 Tg-B and WT mice were similar in the fed state, in the fasted state the transgenic mice had significantly greater plasma glucose than did the control animals (Figure 4A). The serum FFA concentration was also significantly increased in MCP-1 Tg-B mice compared with control mice. The plasma concentration of insulin and the serum concentrations of triglyceride, cholesterol, and adiponectin did not differ between the 2 genotypes (Figure 4A). The transgenic animals manifested both insulin resistance and glucose intolerance in insulin and glucose tolerance tests, respectively (Figure 4B). Hyperinsulinemic-euglycemic clamp analysis also revealed
MCP-1 Tg-B mice to be insulin resistant: the glucose infusion rate was reduced by $54 \%$ in the transgenic mice compared with the WT controls (Figure 4C). The rate of glucose disappearance was reduced by $21 \%$, basal hepatic glucose production was not significantly altered $(P=0.07)$, and hepatic glucose production during the clamp period was markedly increased in MCP-1 Tg-B mice compared with control mice. The ability of insulin to suppress basal hepatic glucose production was thus reduced in MCP-1 Tg-B mice compared with WT mice (20\% versus $75 \%$ suppression). These results were indicative of insulin resistance in both skeletal muscle and liver of MCP-1 Tg-B mice. In addition, the hepatic triglyceride content was increased significantly in MCP-1 Tg-B mice compared with WT mice (Figure 4D). 
A

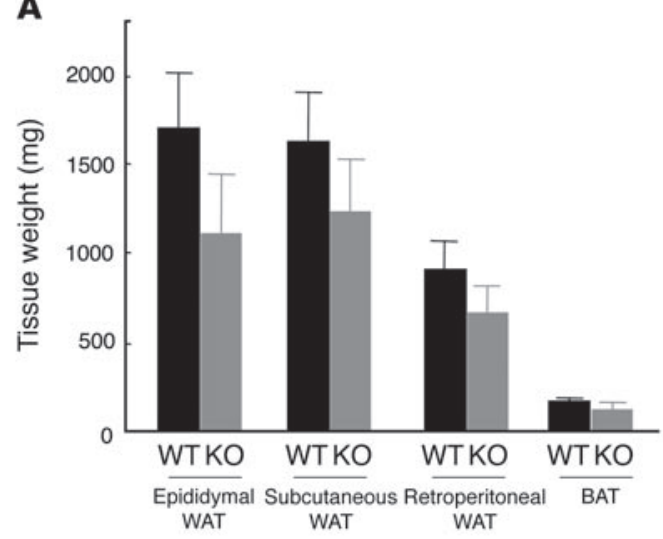

C

WT

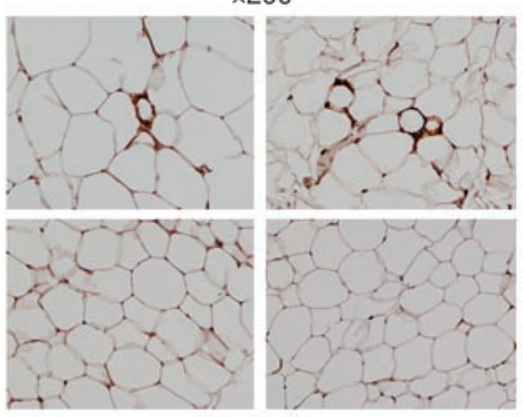

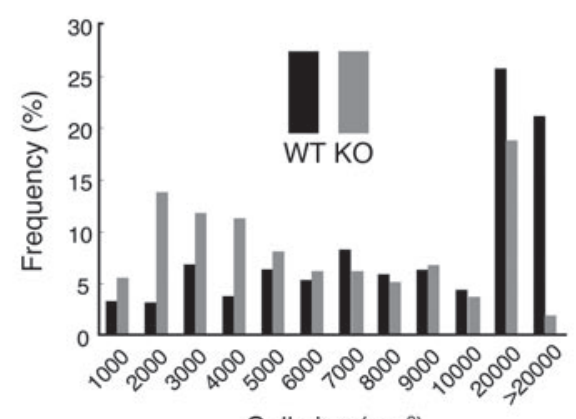

Cell size $\left(\mu \mathrm{m}^{2}\right)$
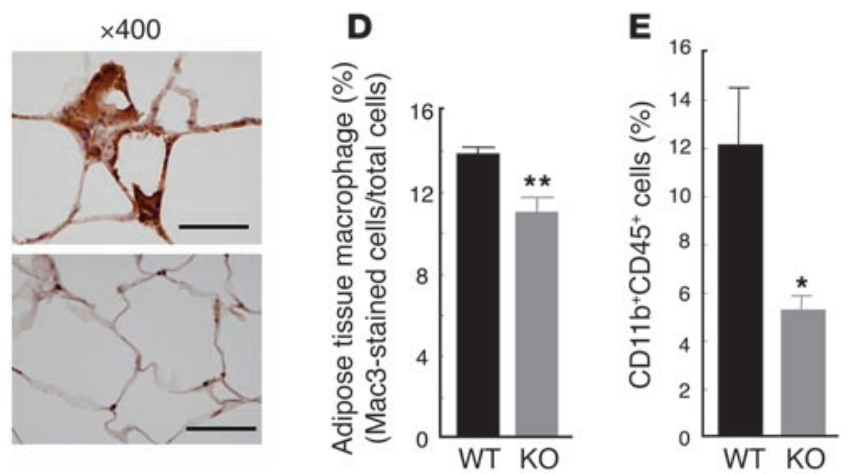

F
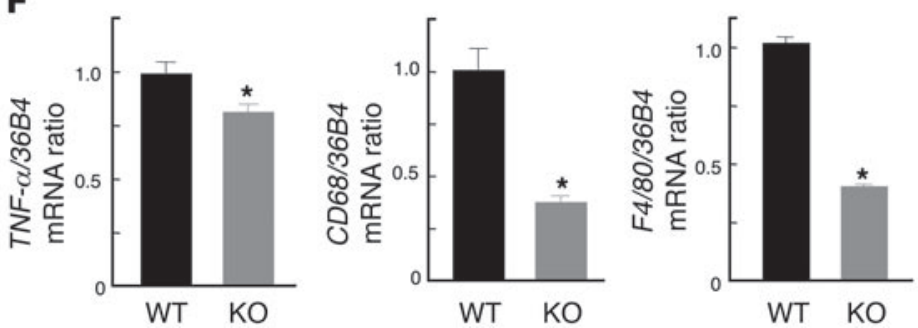

\section{Figure 5}

Characterization of adipose tissue of MCP-1 homozygous KO mice fed a high-fat diet. (A) Weight of various white and brown adipose tissues from the KO and WT mice fed a high-fat diet from 12 to 24 weeks of age. Data are mean \pm SEM $(n=5)$. (B) Size distribution of adipocytes in epididymal fat tissue of mice fed a high-fat diet from 12 to 24 weeks of age. Data are means from analysis of 5 sections from each of 5 mice. (C) Immunohistochemical detection of Mac3 in epididymal adipose tissue of mice fed a high-fat diet from 12 to 24 weeks of age. Magnification, $\times 200$ and $\times 400$, as indicated. Scale bars: $50 \mu \mathrm{m}$. (D) Macrophage infiltration into epididymal fat tissue. Data are mean \pm SEM (WT, $n=8$; KO, $n=6$ ). (E) Quantitation by flow cytometry of the proportion of macrophages in the SVF of epididymal fat tissue from mice fed a high-fat diet from 12 to 28 weeks of age. Data are mean \pm SEM (WT, $n=4 ; \mathrm{KO}, n=6)$. (F) Abundance of macrophage-related protein mRNAs in epididymal fat tissue of mice fed a high-fat diet from 12 to 24 weeks of age. Data (mean $\pm \mathrm{SEM} ; n=4$ ) were normalized by the amount of $36 B 4 \mathrm{mRNA}$ and expressed relative to the corresponding WT value. ${ }^{\star} P<0.05,{ }^{\star \star} P<0.01$ versus WT.

To investigate the mechanism of hepatic insulin resistance and steatosis in MCP-1 Tg-B mice, we first examined expression of the genes for the gluconeogenic enzymes phosphoenolpyruvate carboxykinase (PEPCK) and glucose-6-phosphatase (G6Pase) in the liver after the hyperinsulinemic-euglycemic clamp study. Both of these enzymes are important determinants of hepatic glucose production. Northern blot analysis revealed that after insulin infusion in the fasted mice, the abundance of PEPCK and G6Pase mRNAs was higher in the livers of MCP-1 Tg-B mice than in those of WT mice, although this effect was statistically significant only for G6Pase mRNA (Figure 4E). The amount of the mRNA for SREBP-1c, a transcription factor that regulates the expression of genes important in lipid synthesis, was also significantly increased in the livers of MCP-1
Tg-B mice compared with WT controls after the clamp (Figure 4E). Together, these results indicated that overexpression of MCP-1 in adipocytes results in an increase in the plasma MCP-1 concentration similar to that apparent in $\mathrm{db} / \mathrm{db}$ mice or in mice with obesity induced by a high-fat diet and is sufficient to trigger macrophage infiltration into adipose tissue, insulin resistance, and an increase in hepatic triglyceride content without concomitant obesity.

Reduced ability of a bigh-fat diet to induce insulin resistance and hepatic steatosis in MCP-1 KO mice. Given that the obesity-associated increase in MCP-1 expression was largely restricted to adipose tissue (Figure 2, A and C), we next examined MCP-1 homozygous KO mice to investigate whether the upregulation of MCP- 1 in adipose tissue contributes to obesity-induced insulin resistance. The body 

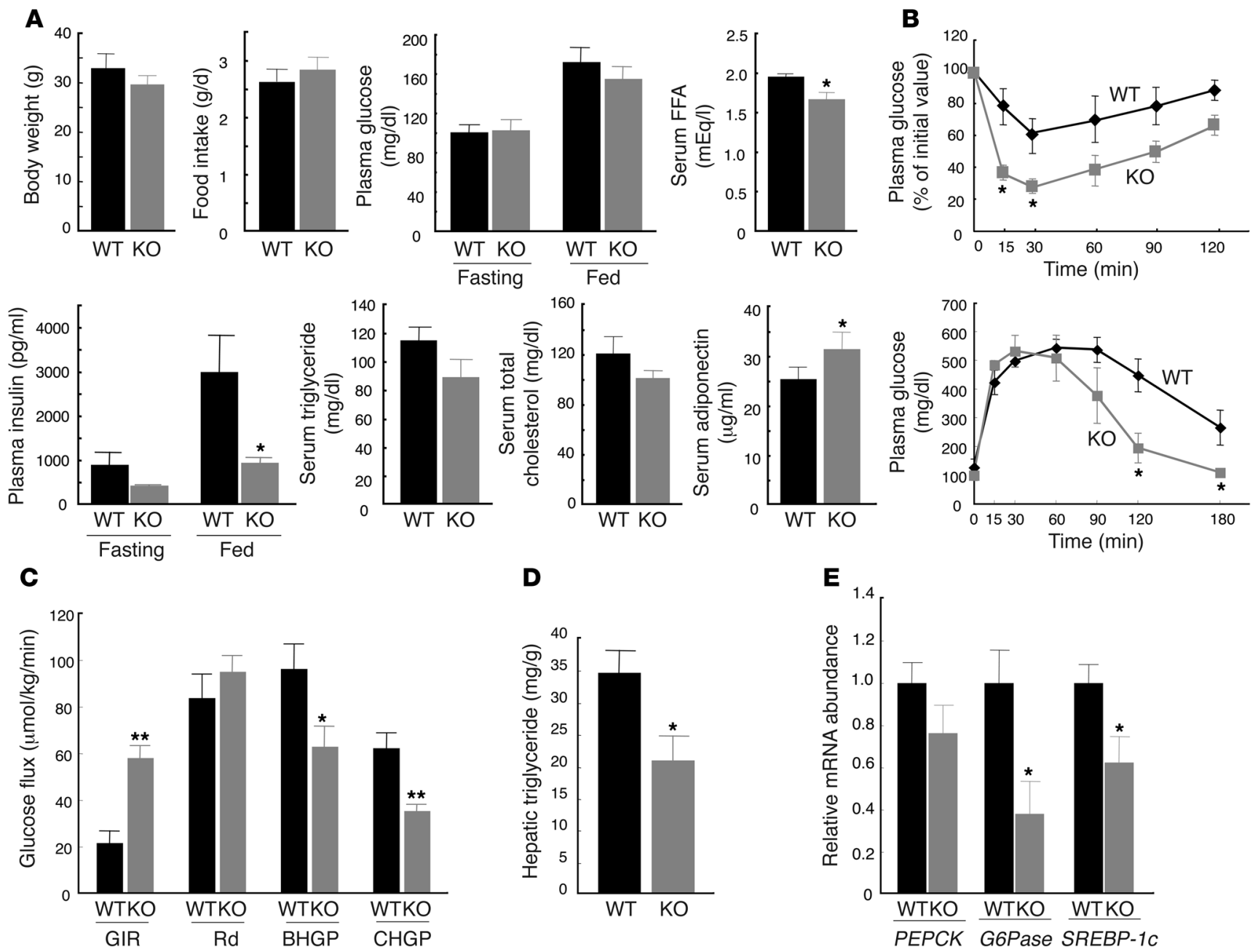

Figure 6

Metabolic characteristics of MCP-1 KO mice fed a high-fat diet. (A) Metabolic parameters of mice fed a high-fat diet from 12 to 24 weeks of age. Data are means \pm SEM (body weight, $n=5$ [WT], 9 [KO]; food intake, $n=4$; plasma glucose, $n=5$; serum FFA, $n=6[\mathrm{WT}], 5$ [KO]; plasma insulin, $n=7$ [WT], 6 [KO]; serum triglyceride, $n=7$ [WT], 6 [KO]; serum total cholesterol, $n=6$ [WT], 5 [KO]; serum adiponectin, $n=7$ [WT], 5 [KO]). (B) Plasma glucose level during insulin (upper panel) and glucose (lower panel) tolerance tests in mice fed a high-fat diet from 12 to 24 weeks of age. Data are mean \pm SEM $(n=6)$. (C) Hyperinsulinemic-euglycemic clamp analysis in mice fed a high-fat diet from 12 to 28 weeks of age. Data are mean \pm SEM (WT, $n=6$; KO, $n=5$ ). (D) Hepatic triglyceride content of mice fed a high-fat diet from 12 to 28 weeks of age. Data are mean \pm SEM $(n=5)$. (E) Quantitation by Northern blot of the hepatic abundance of PEPCK, G6Pase, and SREBP-1C mRNAs in mice after hyperinsulinemic-euglycemic clamp analysis. Data (mean \pm SEM; $n=5$ ) were expressed relative to the corresponding WT value. ${ }^{\star} P<0.05,{ }^{\star \star} P<0.01$ versus WT.

weight of MCP-1 homozygous KO mice fed normal chow did not differ significantly from that of WT control mice at 24 weeks of age $(27.1 \pm 0.82 \mathrm{~g}$ versus $27.6 \pm 0.58 \mathrm{~g}$, respectively; $n=5)$. Adipose tissue weight, adipocyte size, insulin tolerance, and glucose tolerance also did not differ significantly between the 2 groups of mice maintained on normal chow (data not shown). These data suggest that MCP- 1 plays a minimal role in glucose metabolism and insulin sensitivity in mice fed a normal diet.

We next characterized MCP-1 homozygous KO mice fed a highfat diet. Maintenance of WT mice on a high-fat diet from 12 to 24 weeks of age resulted in an approximately 2 -fold increase in the plasma concentration of MCP-1 compared with WT mice fed normal chow (normal chow, $55 \pm 13.7 \mathrm{pg} / \mathrm{ml}$; high-fat diet, $98 \pm 13.5 \mathrm{pg} / \mathrm{ml}$; $n=7$ ), similar to the results shown in Figure 2D. The mass of subcutaneous adipose tissue, the epididymal fat pad, and the retro- peritoneal fat pad tended to be smaller in MCP-1 KO mice than in WT mice on the high-fat diet (Figure 5A). Furthermore, the increase in adipocyte size induced by the high-fat diet was less pronounced in MCP-1 KO mice than in control mice (Figure 5B). Immunohistochemical analysis with the $\mathrm{mAb}$ to Mac3 also showed that the macrophage infiltration into adipose tissue induced by the high-fat diet was extensively reduced in MCP-1 KO mice (Figure 5, $\mathrm{C}$ and D). This finding was confirmed by flow cytometry (Figure 5E). The amounts of TNF- $\alpha, C D 68$, and F4/80 mRNAs in epididymal fat tissue were significantly smaller in MCP-1 KO mice on the highfat diet compared with those in WT controls (Figure 5F). These results suggest that MCP-1 is required for the macrophage infiltration into adipose tissue induced by a high-fat diet.

Body weight, food intake, and plasma glucose concentrations did not differ significantly between MCP-1 KO and WT mice maintained 
A

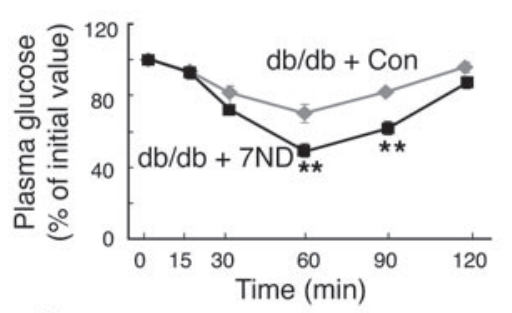

C

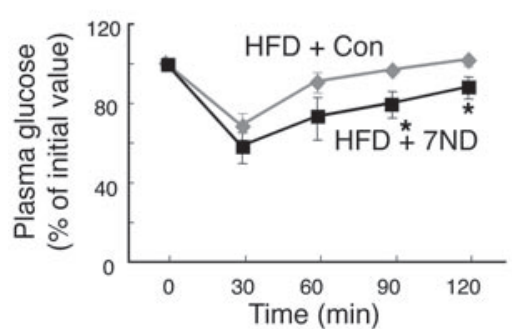

E

$\mathrm{db} / \mathrm{db}+$ Con

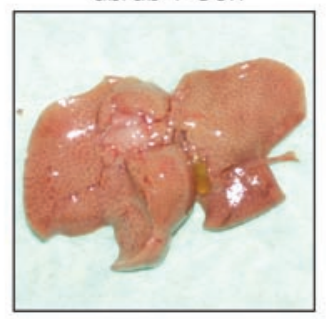

B
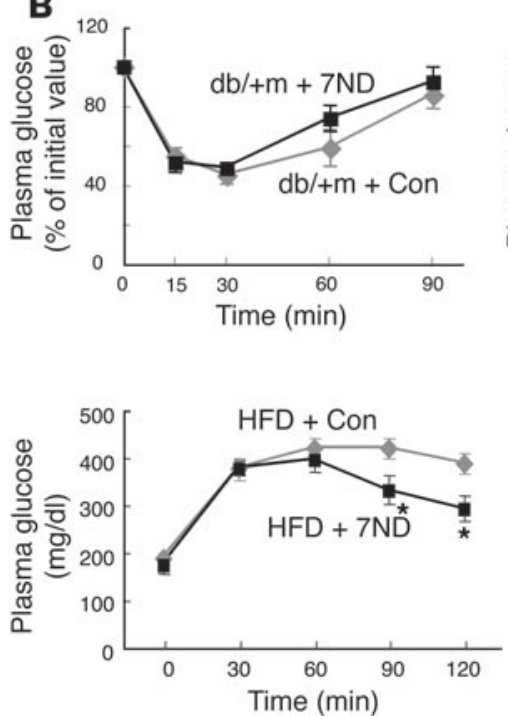

$\mathrm{db} / \mathrm{db}+7 \mathrm{ND}$

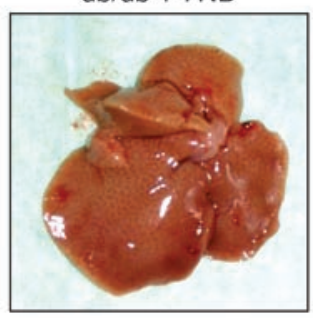

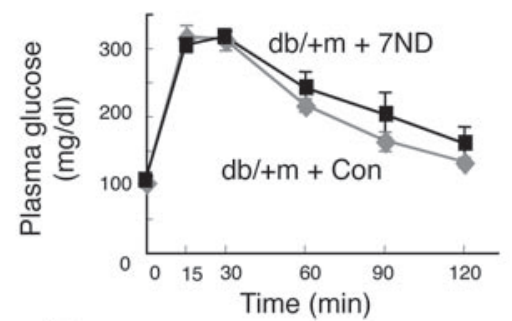

D
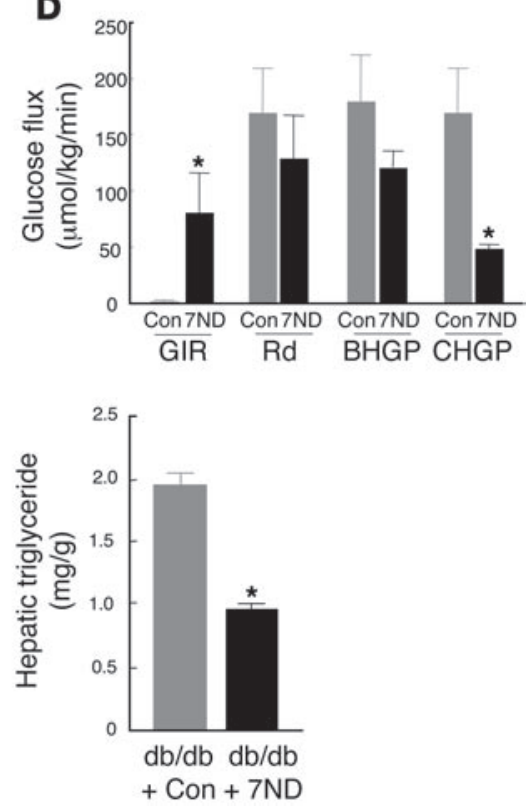

\section{Figure 7}

Effects of expression of the MCP-1 mutant 7ND on insulin sensitivity in obese mice. (A) Insulin tolerance test in db/db mice determined insulin sensitivity 21 days after transfection of 8-week-old animals with the 7ND vector $(n=10)$ or the corresponding empty plasmid as a control (Con, $n=6)$. (B) Insulin (left) and glucose (right) tolerance tests performed 21 days after transfection of 8-week-old db/+m mice with the 7ND vector ( $n=8$ ) or the corresponding empty plasmid $(n=7)$. (C) Insulin (left) and glucose (right) tolerance tests performed 21 days after transfection of 30-week-old C57BL/6J mice fed a high-fat diet since 6 weeks of age with either the 7ND expression vector or the corresponding empty plasmid. Data are mean \pm SEM (insulin tolerance test, $n=4$; glucose tolerance test, $n=5$ [7ND], 7 [empty vector]). (D) Hyperinsulinemic-euglycemic clamp analysis of db/db mice performed 21 days after transfection of 8-week-old animals with the 7ND vector $(n=4)$ or the corresponding empty plasmid $(n=5)$. Data are mean \pm SEM. (E) Appearance of the liver (left) and hepatic triglyceride content $(n=4$, right) in db/db mice 21 days after injection of 8 -week-old animals with the 7ND expression plasmid or the corresponding empty vector. ${ }^{\star} P<0.05,{ }^{\star \star} P<0.01$ versus mice injected with the empty vector.

on the high-fat diet for 12 weeks (Figure 6A). The serum FFA level was significantly decreased in MCP-1 KO mice on the high-fat diet compared with that in WT mice (Figure 6A). The plasma insulin level in the fed state was significantly decreased and the serum adiponectin concentration was significantly increased in MCP-1 KO mice on the high-fat diet compared with those in WT controls (Figure 6A). The insulin resistance and glucose intolerance induced by the high-fat diet in WT mice were both ameliorated in MCP-1 KO mice, as revealed by insulin and glucose tolerance tests, respectively (Figure 6B). Hyperinsulinemic-euglycemic clamp analysis also showed that the glucose infusion rate was increased about 3-fold, both basal and clamp hepatic glucose production were significantly reduced, and the rate of glucose disappearance was unaltered in MCP-1 KO mice (Figure 6C). The MCP-1 KO animals were also protected from the hepatic steatosis apparent in WT mice fed the high-fat diet (Figure 6D). Again, we examined the hepatic expression of PEPCK, G6Pase, and SREBP-1c genes after the clamp study. The abundance of G6Pase and $S R E B P-1 c \mathrm{mRNAs}$ decreased significantly, and PEPCK mRNA tended to be reduced, in MCP-1 KO mice compared with control animals (Figure 6E), suggesting that MCP-1 regulates the hepatic expression of the corresponding genes either directly or indirectly. These results suggest that MCP-1 is important in the pathogenesis of the macrophage infiltration into adipose tissue, insulin resistance, and hepatic steatosis induced by a high-fat diet.

Effects of inbibition of endogenous MCP-1 function by expression of a dominant-negative mutant in vivo. MCP-1 homozygous $\mathrm{KO}$ mice lack MCP-1 throughout embryonic and adult life. To examine whether acute loss of MCP-1 function also results in a protective effect against obesity-induced insulin resistance, we inhibited MCP-1 activity in vivo by inducing the expression of a dominant-negative MCP-1 mutant. A mutant of MCP-1 that lacks the aminoterminal 7 amino acids was previously shown to act in a dominant-negative manner $(23,24)$. Expression of this mutant of human MCP-1, designated $7 \mathrm{ND}$, was also shown to reduce the extents of both atherogenesis in hypercholesterolemic mice (25) and neointimal hyperplasia after periarterial injury in mice and monkeys (26). 
An expression plasmid encoding the 7ND mutant or the corresponding empty vector was injected into the femoral muscle of mice, and the muscle was then subjected to electric pulses from a generator. Muscle treated in this manner has been shown to express the 7ND protein and to secrete it into the bloodstream (25), and the plasma concentration of 7ND was found to be maintained for at least 21 days after transfection (26). In $\mathrm{db} / \mathrm{db}$ mice, the plasma concentration of $7 \mathrm{ND}$, determined with an immunoassay specific for human MCP-1, was $1,140.2 \pm 211.7 \mathrm{pg} / \mathrm{ml}$ and $211.7 \pm 73.6 \mathrm{pg} / \mathrm{ml}(n=6)$ on days 5 and 21 after transfection, respectively. The 7ND mutant was previously shown to form inactive heterodimers with endogenous mouse MCP-1 (24). Given that the plasma concentration of endogenous MCP- 1 in $\mathrm{db} / \mathrm{db}$ mice is approximately $80 \mathrm{pg} / \mathrm{ml}$ (Figure $2 \mathrm{~B}$ ), $7 \mathrm{ND}$ likely inhibited endogenous MCP-1 function in these animals for at least 21 days after transfection. We thus performed metabolic assays in such mice 21 to 23 days after transfection.

Expression of 7ND in $\mathrm{db} / \mathrm{db}$ mice did not affect body weight, food intake, plasma glucose or insulin levels in the fasting or fed state, or the serum adiponectin concentration (data not shown). In contrast, expression of 7ND ameliorated insulin resistance in $\mathrm{db} / \mathrm{db}$ mice compared with $\mathrm{db} / \mathrm{db}$ animals injected with the empty vector (Figure 7A). We did not perform a glucose tolerance test in $\mathrm{db} / \mathrm{db}$ mice because of the high level of plasma glucose in these animals without glucose loading. Expression of 7ND affected neither insulin sensitivity nor glucose tolerance in $\mathrm{db} /+\mathrm{m}$ mice (Figure $7 \mathrm{~B}$ ). We next examined the effects of 7ND expression in mice with high-fat dietinduced obesity. Expression of 7ND resulted in a significant ( $20 \%)$ decrease in the plasma glucose concentration of these animals in the fed state, but it had no effect on body weight, food intake, plasma insulin level in the fed state, fasting plasma glucose concentration, or serum adiponectin level (data not shown). Furthermore, expression of 7ND significantly increased both insulin sensitivity and glucose tolerance in mice with obesity induced by a high-fat diet (Figure 7C). Hyperinsulinemic-euglycemic clamp analysis also revealed that the glucose infusion rate was greatly increased and clamp hepatic glucose production was significantly decreased in $\mathrm{db} / \mathrm{db}$ mice expressing 7ND compared with $\mathrm{db} / \mathrm{db}$ mice transfected with the empty vector (Figure 7D). These latter results for $\mathrm{db} / \mathrm{db}$ mice expressing 7ND were similar to those obtained with MCP-1 KO mice fed a high-fat diet (Figure 6C), suggesting that increased production of MCP-1 in obesity contributes to increased hepatic glucose production. In addition, 7ND expression reduced the hepatic triglyceride content of $\mathrm{db} / \mathrm{db}$ mice by approximately $50 \%$ (Figure $7 \mathrm{E}$ ). These results suggest that inhibition of increased MCP-1 activity after the development of obesity by expression of a dominant-negative mutant of MCP-1 ameliorated insulin resistance and hepatic steatosis.

\section{Discussion}

Previous studies have suggested that MCP-1 is an adipokine whose expression is increased in obese animals $(11,15,16,20)$. However, it has remained unclear whether MCP-1 contributes to development of the insulin resistance and hepatic steatosis associated with obesity. We have now shown that expression of an MCP-1 transgene in adipose tissue under the control of the $a P 2$ gene promoter was sufficient to induce macrophage infiltration into adipose tissue, insulin resistance, and increased hepatic triglyceride content in mice. Furthermore, disruption of the $M C P-1$ gene reduced the extents of macrophage accumulation in adipose tissue, insulin resistance, and hepatic steatosis associated with obesity. In addition, acute inhibition of MCP-1 by expression of a dominant-negative mutant also ameliorated insulin resistance and hepatic steatosis in obese mice. Our results indicate that the increased expression of MCP-1 in adipose tissue that is associated with obesity plays an important role in the pathogenesis of insulin resistance, macrophage infiltration into adipose tissue, and hepatic steatosis.

We found that the MCP-1 gene is expressed in 3T3-L1 adipocytes and in adipocytes of obese mice. Furthermore, macrophage infiltration was apparent in adipose tissue of transgenic mice that overexpress MCP-1 in adipocytes. In contrast, the macrophage accumulation in adipose tissue induced by feeding on a high-fat diet in normal mice was inhibited in MCP-1 homozygous $\mathrm{KO}$ mice. These results suggest that secretion of MCP-1 from adipocytes directly triggers the recruitment of macrophages to adipose tissue. The infiltrated macrophages may in turn secrete a variety of chemokines and other cytokines that further promote a local inflammatory response and affect gene expression in adipocytes, resulting in systemic insulin resistance. However, given that 3 types of transgene controlled by the $\mathrm{aP} 2$ gene promoter were shown to be expressed in macrophages of mice (27), we cannot exclude the possibility that the MCP-1 transgene was expressed in macrophages of our MCP-1 Tg-B mice and that the MCP-1 produced by these cells contributed to the infiltration of macrophages into the adipose tissue of these animals. Nevertheless, the observation that MCP-1 $\mathrm{Tg}-\mathrm{B}$ mice manifest a similar phenotype to obese mice in terms of insulin sensitivity and hepatic steatosis suggests that MCP-1 expression in adipose tissue plays an important role in the insulin resistance and hepatic steatosis associated with obesity.

During the preparation of this manuscript, a report was published that high-fat feeding-induced obesity, adipose tissue macrophage infiltration, adipose tissue inflammation, systemic insulin resistance, and hepatic steatosis were decreased in CCR2 KO mice (28). These phenotypes of CCR2 KO mice were very similar to those of our MCP-1 KO mice, except for adipocyte size, which was larger in CCR2 $\mathrm{KO}$ mice than in controls in high-fat diet feeding. The reason for this difference in adipocyte size was not clear. Complex and redundant interactions are known between chemokines and their receptors. CCR2 also recognizes MCP-2 (29), MCP-3 (30), and MCP-4 (31) as well as MCP-1. On the other hand, MCP-1 is the strongest ligand for CCR2 (32), although it also shows low affinity for CCR11 (33). The similar phenotypes obtained with MCP-1 and CCR2 KO mice suggest that the MCP-1-CCR2 pathway is actually important for obesity-induced insulin resistance and hepatic steatosis among these redundant chemokine signaling pathways.

Our results obtained with MCP-1 transgenic mice, MCP-1 homozygous $\mathrm{KO}$ mice fed a high-fat diet, and obese mice expressing a dominant-negative mutant of MCP-1 support the notion that MCP-1 is responsible for the hepatic insulin resistance and steatosis observed in MCP-1 transgenic mice and obese mice. The increased hepatic glucose production and steatosis apparent in these animals may be explained at least in part by the associated changes in hepatic mRNA levels both for PEPCK and G6Pase, key enzymes of gluconeogenesis, and for SREBP-1c, an important transcription factor for lipid synthesis. Given that hepatic expression of MCP-1 and infiltration of macrophages into the liver were not detected in MCP-1 Tg-B mice and that the MCP-1 receptor CCR2 is expressed in the liver (34), MCP-1 secreted from adipose tissue into the circulation of MCP-1 Tg-B mice may increase the hepatic expression of PEPCK, G6Pase, and SREBP-1c genes by interacting with CCR2 in the liver. Furthermore, given that hepatic steatosis is frequently associated with hepatic insulin resistance, the increase 
in the abundance of SREBP-1c mRNA in the liver may be responsible for the increases in PEPCK and G6Pase gene expression. However, overexpression of SREBP-1c was previously found to suppress the hepatic expression of PEPCK and G6Pase genes both in vitro and in vivo (35), suggesting that MCP-1 may increase the abundance of SREBP-1c mRNA and that of PEPCK and G6Pase mRNAs by independent mechanisms. The precise mechanisms by which MCP-1 induces hepatic insulin resistance and steatosis require further investigation. Serum FFA levels were significantly increased in MCP-1 Tg-B mice and decreased in MCP-1 KO mice fed a high-fat diet compared with corresponding control animals. These changes in serum FFA level might also contribute to the associated changes in hepatic triglyceride content and insulin resistance (36).

Our results with various mouse models have shown that MCP-1 links obesity to insulin resistance, hepatic steatosis, and macrophage infiltration into adipose tissue. Recent studies have also associated MCP-1 with metabolic state in humans. The abundance of MCP-1 mRNA in subcutaneous adipose tissue was found to correlate significantly with both plasma MCP-1 and BMI, and the plasma MCP-1 level also correlated directly with BMI (37). In addition, the plasma concentration of MCP-1 was significantly associated with markers of the metabolic syndrome, including insulin resistance, type 2 diabetes, hypertension, obesity, waist/hip ratio, and increased serum triglyceride concentration (38). MCP-1 expression is higher in visceral than in subcutaneous human adipose tissue (39), and expression of a macrophage antigen in human subcutaneous adipose tissue was significantly correlated with both BMI and adipocyte size (15). These observations are consistent with the hypothesis that MCP-1 contributes both to the recruitment of macrophages to adipose tissue and to the development of insulin resistance in humans through a mechanism similar to that shown by the present study to be operative in mice. Furthermore, treatment with the insulin-sensitizing agent rosiglitazone resulted in a reduction in plasma MCP-1 concentration in both obese nondiabetic and obese diabetic individuals (40). We have also shown that administration of troglitazone to mice with obesity induced by a high-fat diet inhibited both the upregulation of $M C P-1$ gene expression in adipose tissue and the infiltration of macrophages into adipose tissue ( $\mathrm{H}$. Kanda, unpublished observations). Given that PPAR $\gamma$ is expressed in mature macrophages (41), it is possible that thiazolidinediones reduce the plasma concentration of MCP-1 by inhibiting MCP-1 expression in macrophages recruited to adipose tissue.

Obesity, especially visceral obesity, is an important feature of metabolic syndrome, which is also characterized by insulin resistance, glucose intolerance, dyslipidemia, and hypertension. Our present data suggest that MCP-1 links obesity and insulin resistance through induction of an inflammatory response in adipose tissue. We have shown that inhibition of MCP-1 function ameliorated both insulin resistance and hepatic steatosis as well as reduced the extent of macrophage infiltration into adipose tissue of obese mice. Our results thus suggest that MCP-1 plays an important role in the pathogenesis of metabolic syndrome and that inhibition of the interaction of MCP-1 with CCR2 might provide the basis for development of new therapies for this syndrome.

\section{Methods}

Oligonucleotide microarray analysis. 3T3-L1 adipocytes cultured for 10 days after the induction of differentiation were incubated for 36 hours in DMEM containing either glucose $(450 \mathrm{mg} / \mathrm{dl})$ or sucrose $(840 \mathrm{mg} / \mathrm{dl})$, with replenishment of the medium every 6 hours. Alternatively, the cells were cultured for 36 hours in DMEM, some supplemented with $5 \mu \mathrm{M}$ rosiglitazone. Total RNA was prepared from the cells with an RNeasy mini kit (QIAGEN), and portions $(10 \mu \mathrm{g})$ were used for the synthesis of biotin-labeled cRNA, which in turn was used to probe GeneChip mouse microarrays (MGU74.1; Affymetrix). After washing and staining, the arrays were scanned with a Hewlett Packard confocal laser scanner and visualized with Affymetrix GeneChip 3.1 software. The results were analyzed with GeneChip Analysis Suite software (version 4.0; Affymetrix), and the fold differences in hybridization intensity between samples from cells cultured with or without glucose and with or without rosiglitazone were determined.

Experimental animals. C57BL/6J mice, C57BL/6N mice, $\mathrm{db} / \mathrm{db}$ mice, and $\mathrm{db} /+\mathrm{m}$ mice were obtained from CLEA Japan Inc., and MCP-1 KO mice were from The Jackson Laboratory. A high-fat diet (56\% of calories from fat) was from Oriental Yeast Co. Ltd.

For generation of mice that overexpress MCP-1 in adipose tissue, the coding region of mouse MCP- 1 cDNA was obtained by RT-PCR from total RNA extracted from epididymal adipose tissue of $\mathrm{C} 57 \mathrm{BL} / 6 \mathrm{~J}$ mice, and its sequence was confirmed by sequencing of the PCR product. The intron of the rabbit $\beta$-globin gene and a polyadenylation signal from the human growth hormone cDNA (both kindly provided by J. Miyazaki, Osaka University Graduate School of Medicine, Osaka, Japan) were ligated to the $5^{\prime}$ and $3^{\prime}$ flanking regions of the $M C P-1$ coding sequence, respectively. The resulting DNA fragment was then positioned downstream of a $5.4-\mathrm{kb}$ promoter-enhancer fragment of the mouse aP2 gene (kindly provided by B.M. Spiegelman, DanaFarber Cancer Institute, Boston, Massachusetts, USA) (22). The fusion construct composed of the mouse aP2 promoter-enhancer, rabbit $\beta$-globin intron, mouse $M C P-1$ coding sequence, and polyadenylation signal was injected into the pronucleus of fertilized C57BL/6N mouse eggs. The viable injected eggs were transferred to pseudopregnant CD-I female mice (Charles River Laboratories). Transgenic founder mice were identified by Southern blot analysis of tail DNA with the mouse MCP-1 cDNA fragment as a probe.

For $7 \mathrm{ND}$ expression experiments, $\mathrm{db} / \mathrm{db}$ and $\mathrm{db} /+\mathrm{m}$ mice received the transgene at 8 weeks of age and were subjected to metabolic assays at 11 weeks of age. C57BL/6J mice were fed with a high-fat diet from 6 weeks of age, received the transgene at 30 weeks, and were subjected to metabolic assays at 33 weeks.

All mice were maintained under a 12-hour light, 12-hour dark cycle and had access to food and water ad libitum unless indicated otherwise. All studied mice were males. All experimental protocols with mice were approved by the animal ethics committee of Kobe University Graduate School of Medicine.

Transfection of skeletal muscle in situ. An expression plasmid for the dominant-negative mutant of human MCP-1 (7ND) was constructed from pcDNA3 as described previously (24). Mice were anesthetized by injection of ketamine hydrochloride ( $30 \mathrm{mg} / \mathrm{kg}$ body mass i.p.), and femoral muscle of the left leg was exposed. Either pcDNA3-7ND or the corresponding empty vector (100 $\mu \mathrm{g}$ DNA in $50 \mu \mathrm{l} 10 \mathrm{mM}$ Tris-EDTA buffer, $\mathrm{pH}$ 8.0) was injected 4 times into the exposed femoral muscle with a 27 -gauge needle. A pair of electrode needles (BEX) spaced $5 \mathrm{~mm}$ apart was then immediately inserted into the muscle bed on either side of the injection sites, and 3 square-wave pulses ( $100 \mathrm{~V}$ for $100 \mathrm{~ms}$ ) were applied with the use of an electric pulse generator (CUY21; BEX), first with one polarity and then with the opposite polarity. The wound was then closed. No inflammation was observed at the site of surgery in any animal.

Isolation of the SVF and adipocyte fraction of adipose tissue. Mouse epididymal fat pads were minced and digested for 30 minutes at $37^{\circ} \mathrm{C}$ with type 1 collagenase ( $4 \mathrm{mg} / \mathrm{ml}$; Worthington Biochemical Corp.) in DMEM containing $5 \%$ FBS ( $\mathrm{pH} 7.4$ ). After the addition of 3 vol PBS containing $5 \%$ FBS and filtration of the digested tissue through a nylon mesh $(100 \mu \mathrm{m})$, the filtrate was centrifuged at $200 \mathrm{~g}$. The SVF and adipocyte fraction were obtained from the resulting pellet and supernatant, respectively (42). 
Isolation of total RNA, Northern blot analysis, and real-time PCR. Total RNA was extracted from various tissues with the use of TRIzol (Invitrogen Corp.), and portions $(10-15 \mu \mathrm{g})$ of the isolated RNA were subjected to Northern blot analysis. The probe for mouse $M C P-1$ mRNA was obtained by RT-PCR from total RNA extracted from 3T3-L1 adipocytes. Probes for PEPCK, G6Pase, and SREBP-1 mRNAs were kindly provided by D.K. Granner (Vanderbilt University School of Medicine, Nashville, Tennessee, USA), H. Nakajima (Osaka Medical Center for Cancer and Cardiovascular Diseases, Osaka, Japan), and H. Shimano (University of Tsukuba, Ibaraki, Japan), respectively. All probes were labeled with ${ }^{32} \mathrm{P}$ with the use of a Rediprime II random-primer labeling system (Amersham Biosciences). Autoradiograms of blots were visualized with a BAS2500 image analyzer (Fuji Photo Film Co. Ltd.). The SREBP-1 probe reacts with both SREBP-1 $a$ and SREBP-1c mRNAs; however, given that SREBP-1c is the major SREBP-1 isoform in liver, the hepatic signal generated by this probe was assumed to reflect the abundance of SREBP-1c mRNA.

For real-time quantitative PCR analysis, cDNA synthesized from total RNA was analyzed in a Sequence Detector (model 7900; Applied Biosystems) with specific primers and SYBR Green PCR Master reagents (PerkinElmer). The relative abundance of mRNAs was calculated with $36 B 4$ mRNA as the invariant control. The primers were as follows: mouse TNF- $\alpha$, sense, $5^{\prime}$ CCCACACCGTCAGCCGATTT-3', antisense 5'-GTCTAAGTACTTGGGCAGATTGACC-3'; mouse F4/80, sense, 5'-CTTTGGCTATGGGCTTCCAGTC$3^{\prime}$, antisense, 5'-GCAAGGAGGACAGAGTTTATCGTG-3'; mouse CD68, sense, 5'-CTTCCCACAGGCAGCACAG-3', antisense, 5'-AATGATGAGAGGCAGCAAGAGG-3'; and mouse 36B4, sense, 5'-GAGGAATCAGATGAGGATATGGGA-3', antisense, 5'-AAGCAGGCTGACTTGGTTGC-3'.

Flow cytometry. The SVF of mouse epididymal adipose tissue was prepared as described above. Red blood cells present in this fraction were lysed with the use of Pharm Lyse (BD Biosciences), and the remaining cells were suspended in PBS containing $2 \mathrm{mM}$ EDTA and exposed to FcBLOCK (BD Biosciences) for 20 minutes. The cells were then labeled with PE-conjugated antibodies to mouse CD11b (M1/70; BD Biosciences) and FITC-conjugated antibodies to mouse CD45 (BD Biosciences); dead cells were stained with 7-amino-actinomycin D (BD Biosciences). Live cells positive for both $\mathrm{CD} 11 \mathrm{~b}$ and CD45 (macrophages) were then quantitated by flow cytometry with a FACSCalibur analyzer (BD Biosciences) (42).

Analysis of metabolic parameters. The plasma concentration of mouse MCP-1 or human 7ND was measured with Quantikine $\mathrm{M}$ mouse and human MCP-1 ELISA kits (R\&D Systems). Plasma insulin concentration was measured with an insulin assay kit (Morinaga Institute of Biological Science). FFA, cholesterol, and triglyceride concentrations in serum were measured with NEFA-C, Cholesterol E, and Triglyceride E tests (Wako Pure Chemical Industries Inc.), respectively. Serum adiponectin level was measured with an adiponectin ELISA kit (Otsuka Pharmaceutical Co. Ltd.). For glucose tolerance tests, mice were deprived of food for 16-24 hours and then injected i.p. with glucose ( $2 \mathrm{~g} / \mathrm{kg}$ body mass). For insulin tolerance tests, mice were injected i.p. with human regular insulin $(0.6 \mathrm{U} / \mathrm{kg}$ for MCP- $1 \mathrm{Tg}$ mice, $0.75 \mathrm{U} / \mathrm{kg}$ for MCP-1 KO, db/+m, and C57BL/6J mice fed a high-fat diet, and $3.5 \mathrm{U} / \mathrm{kg}$ for $\mathrm{db} / \mathrm{db}$ mice; Eli Lilly and Co.). Blood samples were collected before and after injection, and plasma glucose concentration was measured with an Antisense II instrument (Bayer).

Hyperinsulinemic-englycemic clamp. Hyperinsulinemic-euglycemic clamp analysis was performed as described previously (43), with minor modifications. In brief, 5-7 days before the clamp, mice were anesthetized with sodium pentobarbital ( $80-100 \mathrm{mg} / \mathrm{kg}$ i.p.), and a catheter was inserted into the right internal jugular vein for infusion. The analysis was performed under nonstressful conditions with conscious mice that had been deprived of food overnight for 8 hours (MCP-1 Tg-B and MCP-1 homozygous KO mice) or 16 hours (db/db mice). [3- $\left.{ }^{3} \mathrm{H}\right]$ Glucose was infused for 2 hours at a rate of $0.05 \mu \mathrm{Ci} / \mathrm{min}$ before initiation of the clamp, and a blood sample was collected at the end of this period to estimate basal glucose turnover. After a bolus injection of $\left[3-{ }^{3} \mathrm{H}\right]$ glucose $(10 \mu \mathrm{Ci}$; PerkinElmer $)$ and the onset of subsequent continuous infusion of $\left[3{ }^{3} \mathrm{H}\right]$ glucose $(0.1 \mu \mathrm{Ci} / \mathrm{min})$, a hyperinsulinemic-euglycemic clamp was applied for 120 minutes with continuous infusion of insulin at a rate of $2.5 \mathrm{mU} / \mathrm{kg} / \mathrm{min}$ (for MCP- $1 \mathrm{Tg}-\mathrm{B}$ and MCP-1 $\mathrm{KO}$ mice) or $18 \mathrm{mU} / \mathrm{kg} / \mathrm{min}$ (for $\mathrm{db} / \mathrm{db}$ mice). Plasma glucose concentration was monitored every 10 minutes, and $30 \%$ glucose was infused at a variable rate to maintain plasma glucose at basal concentrations. Blood samples were collected 80, 90,100,110, and 120 minutes after the onset of the clamp for determination of the plasma concentrations of $\left[3-{ }^{3} \mathrm{H}\right]$ glucose and ${ }^{3} \mathrm{H}_{2} \mathrm{O}$. The rates of glucose disposal and hepatic glucose production were calculated as described previously (43).

Measurement of hepatic triglyceride content. For determination of hepatic triglyceride content in MCP-1 Tg-B and MCP-1 homozygous KO mice, liver tissue (100-200 $\mathrm{mg}$ ) was homogenized for 10 minutes in $4 \mathrm{ml}$ isopropanol with a Polytron disrupter. The homogenate was centrifuged at 2,000 $\mathrm{g}$ for 10 minutes, and $10 \mu \mathrm{l}$ of the resulting supernatant were dried with a Speedvac System (Thermo Electron Corp.) (44). The dry residue was dissolved in $5 \mu \mathrm{l}$ isopropanol, and its triglyceride content was measured with a Triglyceride E test (Wako Pure Chemical Industries Ltd.) (45). For $\mathrm{db} / \mathrm{db}$ mice, liver fragments $(50-100 \mathrm{mg}$ ) were subjected to extraction for 16 hours at $4{ }^{\circ} \mathrm{C}$ with $4 \mathrm{ml} \mathrm{CHCl} / 3$ methanol $(2: 1, \mathrm{vol} / \mathrm{vol})$. Two milliliters of $0.6 \% \mathrm{NaCl}$ were added to the extract, and the mixture was centrifuged at 2,000 $\mathrm{g}$ for 20 minutes (46). The organic layer was collected and dried, and the residue was dissolved in isopropanol and assayed for triglyceride content with a Triglyceride E test. All values of tissue triglyceride content were corrected for liver weight.

Histological analysis. Adipose tissue was fixed for 24-48 hours with $10 \%$ paraformaldehyde at $4{ }^{\circ} \mathrm{C}$, dehydrated, embedded in paraffin, and cut into 5 - $\mu \mathrm{m}$-thick sections at $50-\mu \mathrm{m}$ intervals. The sections were mounted on glass slides, depleted of paraffin with xylene, and microwaved (500 W for $5 \mathrm{~min}$ ) in $0.01 \mathrm{M}$ sodium citrate buffer ( $\mathrm{pH}$ 6.0). After blocking of endogenous biotin and avidin binding sites with a Biotin Blocking System (Dako) and blocking of $\mathrm{Fc}$ receptors with PBS containing $20 \% \mathrm{FBS}$, sections were subjected to immunohistochemical staining overnight at $4^{\circ} \mathrm{C}$ with a $1: 100$ dilution of a mouse mAb to Mac3 (M3/84; BD Biosciences). Immune complexes were detected with biotinylated secondary antibodies (BD Biosciences), HRP-conjugated streptavidin (Dako), and the peroxidase substrate diaminobenzidine (Dako). After staining with hematoxylin (Muto Pure Chemicals), mounting solution (Matsunami Glass) and cover slips were added to the sections. Slides were observed with a light microscope. Macrophage infiltration in adipose tissue was quantitated by calculating the ratio of nuclei of Mac3-positive cells to total nuclei in 20 fields of 3 slides for each individual mice using 6-9 mice for each group $(15,28)$. Macrophage infiltration into liver and skeletal muscle was estimated by counting the number of Mac3-positive cells in 3 slides prepared from the central portion of each tissue obtained from 5-8 mice. Adipocyte size was measured with the use of MACSCOPE software (Mitani Sangyo Co. Ltd.).

Statistics. Data are presented as means \pm SEM and were analyzed by 2 -tailed Student's $t$ test. A $P$ value $<0.05$ was considered statistically significant.

\section{Acknowledgments}

This work was supported by grants for the Intellectual Cluster Formation Project and the 21st Century COE Program "Center of Excellence for Signal Transduction Disease: Diabetes Mellitus as a Model" from the Ministry of Education, Culture, Sports, Science, and Technology of Japan to M. Kasuga. We thank B.M. Spiegelman for the aP2 promoter-enhancer fragment; J. Miyazaki for the rabbit $\beta$-globin intron and polyadenylation signal from human growth hormone cDNA; D.K. Granner, H. Nakajima, and 
H. Shimano for the PEPCK, G6Pase, and SREBP-1 probes, respectively; and N. Sakai for technical assistance.

Received for publication August 4, 2005, and accepted in revised form March 14, 2006.

Address correspondence to: Yoshikazu Tamori, Division of Diabetes and Digestive and Kidney Diseases, Department of Clinical Molecular Medicine, Kobe University Graduate School of
Medicine, 7-5-1 Kusunoki-cho, Chuo-ku, Kobe 650-0017, Japan. Phone: 81-78-382-5861; Fax: 81-78-382-2080; E-mail: tamori@ med.kobe-u.ac.jp.

Ko Kotani's present address is: Department of Internal Medicine, Kawasaki Medical School, Kurashiki, Japan.

Hajime Kanda and Sanshiro Tateya contributed equally to this work.
1. Spiegelman, B.M., and Flier, J.S. 2001. Obesity and the regulation of energy balance. Cell. 104:531-543.

2. Kahn, B.B., and Flier, J.S. 2001. Obesity and insulin resistance. J. Clin. Invest. 106:473-481.

3. Ahima, R.S., and Flier, J.S. 2000. Adipose tissue as an endocrine organ. Trends Endocrinol. Metab. 11:327-332

4. Yoshimura, T., et al. 1989. Purification and amino acid analysis of two human glioma-derived monocyte chemoattractants. J. Exp. Med. 169:1449-1459.

5. Matsushima, K., Larsen, C.G., DuBois, G.C., and Oppenheim, J.J. 1989. Purification and characterization of a novel monocyte chemotactic and activating factor produced by a human myelomonocytic cell line. J. Exp. Med. 169:1485-1490.

6. Rollins, B.J. 1997. Chemokines. Blood. 90:909-928.

7. Yla-Herttuala, S., et al. 1991. Expression of monocyte chemoattractant protein 1 in macrophage-rich areas of human and rabbit atherosclerotic lesions. Proc. Natl. Acad. Sci. U. S. A. 88:5252-5256.

8. Takeya, M., Yoshimura, T., Leonard, E.J., and Takahashi, K. 1993. Detection of monocyte chemoattractant protein-1 in human atherosclerotic lesions by an anti-monocyte chemoattractant protein-1 monoclonal antibody. Hum. Pathol. 24:534-539.

9. Gu, L., et al. 1998. Absence of monocyte chemoattractant protein-1 reduces atherosclerosis in low density lipoprotein receptor-deficient mice. Mol. Cell. 2:275-281.

10. Boring, M., Gosling, J., Cleary, M., and Charo, I.F. 1998. Decreased lesion formation in CCR2 $2^{-/-}$mice reveals a role for chemokines in the initiation of atherosclerosis. Nature. 394:894-897.

11. Sartipy, P., and Loskutoff, D.J. 2003. Monocyte chemoattractant protein 1 in obesity and insulin resistance. Proc. Natl. Acad. Sci.U. S. A. 100:7265-7270.

12. Hotamisligil, G.S., Shargill, N.S., and Spiegelman, B.M. 1993. Adipose expression of tumor necrosis factor- $\alpha$ : direct role in obesity-linked insulin resistance. Science. 259:87-91.

13. Hotamisligil, G.S., Arner, P., Caro, J.F., Atkinson, R.L., and Spiegelman, B.M. 1995. Increased adipose tissue expression of tumor necrosis factor- $\alpha$ in human obesity and insulin resistance. J. Clin. Invest. 95:2409-2415.

14. Fried, S.K., Bunkin, D.A., and Greenberg, A.S. 1998. Omental and subcutaneous adipose tissues of obese subjects release interleukin-6: depot difference and regulation by glucocorticoid. J. Clin. Endocrinol. Metab. 83:847-850.

15. Weisberg, S.P., et al. 2003. Obesity is associated with macrophage accumulation in adipose tissue. J. Clin. Invest. 112:1796-1808. doi:10.1172/ JCI200319246.

16. Xu, A., et al. 2003. Chronic inflammation in fat plays a crucial role in the development of obesity-related insulin resistance. J. Clin. Invest. 112:1821-1830. doi:10.1172/JCI200319451.

17. Abel, E.D., et al. 2001. Adipose-selective targeting of the GLUT4 gene impairs insulin action in muscle and liver. Nature. 409:729-733.

18. Yang, Q., et al. 2005. Serum retinol binding protein 4 contributes to insulin resistance in obesity and type 2 diabetes. Nature. 436:356-362.

19. Fasshauer, M., et al. 2004. Monocyte chemoattractant protein 1 expression is stimulated by growth hormone and interleukin- 6 in 3T3-L1 adipocytes. Biochem. Biophys. Res. Commun. 317:598-604.

20. Takahashi, K., et al. 2003. Adiposity elevates plasma MCP-1 levels leading to the increased CD11b-positive monocytes in mice. J. Biol. Chem. 278:46654-46660.

21. Coe, N.R., Simpson, M.A., and Bernlohr, D.A. 1999. Targeted disruption of the adipocyte lipid-binding protein (aP2 protein) gene impairs fat cell lipolysis and increases cellular fatty acid levels. J. Lipid Res. 40:967-972.

22. Ross, S.R., et al. 1990. A fat-specific enhancer is the primary determinant of gene expression for adipocyte P2 in vivo. Proc. Natl. Acad. Sci. U. S. A. 87:9590-9594.

23. Zhang, Y.J., Rutledge, B.J., and Rollins, B.J. 1994. Structure/activity analysis of human monocyte chemoattractant protein-1 (MCP-1) by mutagenesis. Identification of a mutated protein that inhibits MCP-1-mediated monocyte chemotaxis. J. Biol. Chem. 269:15918-15924.

24. Zhang, Y., and Rollins, B.J. 1995. A dominant negative inhibitor indicates that monocyte chemoattractant protein 1 functions as a dimer. Mol. Cell. Biol. 15:4851-4855.

25. Ni, W., et al. 2001. New anti-monocyte chemoattractant protein-1 gene therapy attenuates atherosclerosis in apolipoprotein E-knockout mice. Circulation. 103:2096-2101.

26. Egashira, K., et al. 2002. Importance of monocyte chemoattractant protein-1 pathway in neointimal hyperplasia after periarterial injury in mice and monkeys. Circ. Res. 90:1167-1172.

27. Makowski, L., et al. 2001. Lack of macrophage fattyacid-binding protein $\mathrm{aP} 2$ protects mice deficient in apolipoprotein E against atherosclerosis. Nat. Med. 7:699-705

28. Weisberg, S.P., et al. 2006. CCR2 modulates inflammatory and metabolic effects of high-fat feeding. J. Clin. Invest. 116:115-124. doi:10.1172/JCI24335.

29. Gong, X., et al. 1997. Monocyte chemotactic protein-2 (MCP-2) uses CCR1 and CCR2B as its functional receptors. J. Biol. Chem. 272:11682-11685.

30. Combadiere, C., et al. 1995. Monocyte chemoattractant protein-3 is a functional ligand for CC chemokine receptors 1 and 2B. J. Biol. Chem. 270:29671-29675.

31. Garcia-Zepeda, E.A., et al. 1996. Human monocyte chemoattractant protein (MCP)-4 is a novel CC chemokine with activities on monocytes, eosinophils, and basophils induced in allergic and nonallergic inflammation that signals through the CC chemokine receptors (CCR)-2 and -3. J. Immunol. 157:5613-5626.

32. Charo, I. F., et al. 1994. Molecular cloning and func- tional expression of two monocyte chemoattractant protein 1 receptors reveals alternative splicing of the carboxyl-terminal tails. Proc. Natl. Acad. Sci. U. S. A. 91:2752-2756.

33. Schweickart, V.L., Epp, A., Raport, C.J., and Gray, P.W. 2000. CCR11 is a functional receptor for the monocyte chemoattractant protein family of chemokines. J. Biol. Chem. 275:9550-9556.

34. Lu, D., et al. 2005. Cloning and functional characterization of the rabbit C-C chemokine receptor 2 . BMC Immunol. 6:15.

35. Yamamoto, T., et al. 2004. SREBP-1 interacts with hepatocyte nuclear factor- 4 alpha and interferes with PGC-1 recruitment to suppress hepatic gluconeogenic genes. J. Biol. Chem. 279:12027-12035.

36. Lewis, G.F., Carpentier, A., Adeli, K., and Giacca, A. 2002. Disordered fat storage and mobilization in the pathogenesis of insulin resistance and type 2 diabetes. Endocr. Rev. 23:201-229.

37. Christiansen, T., Richelsen, B., and Bruun, J.M. 2005. Monocyte chemoattractant protein- 1 is produced in isolated adipocytes, associated with adiposity and reduced after weight loss in morbid obese subjects. Int. J. Obes. Relat. Metab. Disord. 29:146-150.

38. Simeoni, E., et al. 2004. Association between the $A-2518 G$ polymorphism in the monocyte chemoattractant protein-1 gene and insulin resistance and Type 2 diabetes mellitus. Diabetologia. 47:1574-1580.

39. Bruun, J.M., Lihn, A.S., Pedersen, S.B., and Richelsen, B. 2005. Monocyte chemoattractant protein-1 release is higher in visceral than subcutaneous human adipose tissue (AT): implication of macrophages resident in the AT. J. Clin. Endocrinol. Metab. 90:2282-2289.

40. Mohanty, P., et al. 2004. Evidence for a potent antiinflammatory effect of rosiglitazone. J. Clin. Endocrinol. Metab. 89:2728-2735.

41. Chinetti, G., et al. 1998. Activation of proliferator-activated receptors $\alpha$ and $\gamma$ induces apoptosis of human monocyte-derived macrophages. J. Biol. Chem. 273:25573-25580.

42. Curat, C.A., et al. 2004. From blood monocytes to adipose tissue-resident macrophages: induction of diapedesis by human mature adipocytes. Diabetes. 53:1285-1292.

43. Kim, J.K., et al. 2001. Glucose toxicity and the development of diabetes in mice with muscle-specific inactivation of GLUT4. J. Clin. Invest. 108:153-160. doi:10.1172/JCI200110294.

44. Burant, C.F., et al. 1997. Troglitazone action is independent of adipose tissue. J. Clin. Invest. 100:2900-2908.

45. Tamori, Y., Masugi, J., Nishino, N., and Kasuga, M. 2002. Role of peroxisome proliferator-activated receptor-gamma in maintenance of the characteristics of mature 3T3-L1 adipocytes. Diabetes. 51:2045-2055.

46. Nakamura, K., and Handa, S. 1984. Coomassie brilliant blue staining of lipids on thin-layer plates. Anal. Biochem. 142:406-410. 